\title{
Traduire
}

Une eutre perspective sur $r$ tatadciction

Revue française de la traduction

224 | 2011

Des outils et des nuages

\section{Traduire : un art de la contrainte}

Presses Universitaires de Provence, 2010

\section{Yvon Keromnes}

\section{(2) OpenEdition}

Journals

Édition électronique

URL : http://journals.openedition.org/traduire/235

DOI : 10.4000/traduire.235

ISSN : 2272-9992

Éditeur

Société française des traducteurs

Édition imprimée

Date de publication : 1 juin 2011

Pagination : 135-140

ISSN : 0395-773X

Référence électronique

Yvon Keromnes, «Traduire : un art de la contrainte», Traduire [En ligne], 224 | 2011, mis en ligne le 10 février 2014, consulté le 22 septembre 2020. URL : http://journals.openedition.org/traduire/235 ; DOI : https://doi.org/10.4000/traduire.235 


\section{Compte rendu d'ouvrage Traduire : un art de la contrainte}

\section{Yvon Keromnes}

Cet ouvrage coédité par Charles Zaremba, professeur de linguistique slave, et Noël Dutrait, professeur de langue et littérature chinoises, constitue les Actes d'un colloque consacré à la traduction, organisé par l'Université de Provence et ayant réuni " plus de vingt participants [...] représentant dix-sept langues(1) " (p. 6). À quelques exceptions près (formation des interprètes russophones, terminologie du droit...), il y est question de traduction littéraire et universitaire. Le recueil comprend vingt-deux articles regroupés sous quatre rubriques : Questions générales (quatre articles), Textes et réalités (onze articles), Questions de poésie (quatre articles) et Questions de linguistique (quatre articles également). Y sont présentées des questions touchant à la traduction entre le français et le bulgare, le créole mauricien, l'espagnol, le finnois, le mandarin, le persan, le roumain, le russe, le sanskrit et le tchèque.

Une telle variété de langues et de questions auraient sans doute gagné à être présentées de façon plus didactique, on regrettera notamment sur ce point l'absence d'une véritable introduction. Également regrettable est le nombre important de coquilles qui émaillent le texte.

Voici à présent un aperçu des différents articles.

\section{Questions générales :}

Elena Porshneva (Université de Nijni Novgorod) présente la formation des futurs interprètes russophones à une compétence culturelle française, en particulier à travers l'étude d'œuvres littéraires en français abordant les questions d'interculturalité.

Dans un bref article programmatique, Guennadi Ryabov (Président de l'université de Nijni Novgorod), parle aussi de compétences interculturelles : suite à l'introduction dans le cursus

(1) Et dont on peut apprendre sur internet qu'il a eu lieu en 2008. 
universitaire russe d'un nouveau domaine, Linguistique et communication interculturelle : nouvelles approches didactiques, l'université offre des cours intégrés de littérature, de culture, d'histoire, de religion et d'art pour " mieux comprendre comment les cultures différentes s'interpénètrent et s'enrichissent mutuellement " (p. 21).

Olga Sapojnikova (Université de Nijni Novgorod) propose une méthode de traduction « appelée à rendre la critique de la traduction plus exacte à chaque niveau linguistique en se fondant sur des observations concrètes ", ce qu'elle illustre par quelques exemples tirés d'une traduction de Tchekov publiée en français en 1975, observés d'un point de vue conceptuel, dénotatif, discursif (occasion pour l'auteur de noter que le jeu des registres " est reproduit de manière presque adéquate "), connotatif (dans lequel l'auteur range les realia russes, moujik, etc.) et pragmatique, qui font l'objet de commentaires assez généraux.

\section{Textes et réalités :}

Marie Vrinat-Nikolov (INALCO) citant G. Steiner, pour qui " chaque langue [...] construit un ensemble de mondes possibles et de géographies de la mémoire " (où l'on voit mélangées les notions de langue et de culture), semble embarquer sous la bannière de Sapir et Whorf toute une série de théoriciens de la traduction, dont les conceptions découleraient de la fameuse " idée selon laquelle chaque langue impose une conceptualisation particulière du réel ". Elle en vient aux différences entre le bulgare et le français, avant d'aborder la question de la traduction littéraire, pour laquelle elle plaide en faveur d'un métissage et d'une hybridation.

Inês Oseki-Dépré (Université de Provence) parle surtout de son expérience de la traduction de l'auteur brésilien Joao Guimaraes Rosa en français, dans laquelle, s'inspirant des idées de Berman et de Derrida, elle tâche de rendre compte des spécificités de cet auteur (utilisation particulière des temps verbaux, nombreuses créations lexicales...), spécificités qui sont ensuite en bonne part effacées par des "corrections " de l'éditeur. Faute de contexte, le lecteur est en revanche incapable de juger du "mal fondé " de modifications portant par exemple sur l'ordre des mots ou un temps verbal.

Philippe Che (Université de Provence) présente quelques expressions poétiques et citations plus ou moins allusives chez Ge Hong, auteur du Ive siècle, et la façon dont le traducteur, à force d'érudition, peut résoudre ces questions.

Avec Pierre Kaser (Université de Provence), c'est aussi d'érudition qu'il s'agit pour la traduction d'un auteur chinois dramaturge du XVIIe siècle, Li Yu, sachant que le théâtre littéraire ancien est l'un des domaines les moins traduits de la littérature chinoise, et ce sans doute en premier lieu pour des raisons philologiques.

Noël Dutrait (Université de Provence) parle de son expérience de traduction (en collaboration) d'un roman de l'auteur chinois contemporain Mo Yan (pseudonyme signifiant « ne parle pas »), 
Quarante et un coups de canon. Le narrateur de ce roman est un enfant bavard et affabulateur, et c'est le mélange de rêve et de réalité, de vérité et de mensonge, qui a constitué la difficulté principale de cette traduction. Celle-ci a été facilitée par une correspondance intense avec l'auteur.

Pierre Baccheretti (Université de Provence), à partir de nombreux exemples fort éclairants, expose les difficultés rencontrées à différents niveaux lors de sa traduction en russe de $L a$ gloire de mon père, de Marcel Pagnol. Le projet devait constituer la base d'un manuel de thème à destination des étudiants de russe.

Mirka Ševčiková (Brno) est traductrice tchèque d'Antoine Volodine, auteur qui entend lui-même donner dans ses textes l'impression d'une littérature traduite. Sont détaillés dans cet article les moyens lexicaux et syntaxiques mis en œuvre à cette fin, et les ressources de la traductrice pour obtenir une étrangeté tchèque... qui ne manque pas de lui créer des ennuis auprès de sa maison d'édition.

Milena Fučiková (Université de Provence), traductrice tchèque de Patrick Chamoiseau, pose la nécessité d'une réflexion théorique particulière aux problèmes posés par la traduction du créole en tchèque. S'appuyant sur les propres écrits que l'auteur a adressés à ses traducteurs, elle assume une certaine opacité avec, en particulier, un recours important à des emprunts, mais la dimension re-création est présente aussi, par exemple lorsqu'elle explique comment elle utilise les ressources aspectuelles du tchèque pour créer des expressions correspondant à des particularités du créole (verbes sériels), et comment rendre les jeux de mots ou la musicalité de la langue.

Agnieszka Grudzińska (Université Paris IV) parle de la traduction des Lettres de la Shoa (archives du ghetto de Varsovie), textes reflétant un univers linguistique multiple où le polonais se mélange à l'hébreu et au yiddish (mais on pourra s'interroger sur sa définition du yiddish comme "forme ludique de l'hébreu "), et écrits, on l'imagine, dans des circonstances d'urgence et de grande précarité. On peut estimer que tous les écarts linguistiques, jusqu'aux fautes d'orthographe et de style, font partie de ces documents authentiques et ne devraient pas être gommés, ce qui a malheureusement été fait dans la traduction publiée.

Agnieszka Żuk (Nancy 2) a traduit en collaboration le roman d'un auteur polonais, Andrzej Stasiuk, lequel décrit des scènes avec beaucoup d'économie, esquissant par exemple le tableau d'une famille à la campagne attendant le car dans un abri : le lecteur polonais reconnaît dans ce tableau une famille campagnarde catholique traditionnelle. Mais outre qu'une telle économie descriptive n'est pas nouvelle et que l'on peut laisser au lecteur le soin d'identifier ou non l'implicite, la traductrice exagère peut-être parfois les difficultés par méconnaissance de la réalité française.

Kira Kachliavik (Université de Nijni Novgorod) retrace l'édition des Pensées de Pascal en langue russe, et finit sur la traduction de J.-A. Ginzburg : " le texte presque impeccable au point de vue linguistique, ce que permet (sic) d'avancer sur la voie de la recherche du vrai Pascal „ p. 175. 


\section{Questions de poésie :}

Perle Abbrugiati (Université de Provence) s'intéresse à trois traductions en italien d'une chanson de G. Brassens prise comme "représentatif du texte poétique " (p. 180). Elle observe, parmi les contraintes, que "le texte d'une chanson est destiné à être chanté " (ibid.). De ces contraintes, il ressort que la traduction la plus aboutie formellement est davantage une réélaboration du texte original qu'un rendu fidèle.

Françoise Douay Soublin (Université de Provence) présente un projet de l'atelier « Métaphores polyglottes " avec la participation de neuf traducteurs et dans lequel sont présentées des traductions déjà existantes et des traductions originales d'un poème de Baudelaire. Le projet est " d'étudier avec plus de précision qu'on ne l'a fait jusqu'ici la manière dont se construisent, dans les différentes langues du monde, les métaphores ". Les traductions existantes sont en anglais, les originales sont en créole mauricien, espagnol, roumain, finnois, mandarin, persan, sanskrit. C'est un projet tout à fait intéressant, on regrettera simplement que toutes les traductions ne soient pas accompagnées d'un mot-à-mot.

Pierre Larcher (Université de Provence et IREMAM) discute de l'intérêt de retraduire une anthologie de sept poèmes arabes pré-islamistes : c'est qu'une traduction belle et fidèle reste à faire. II donne en exemple quelques vers de son cru, accompagnés de commentaires tout à fait intéressants, mais l'article s'arrête de façon abrupte sur une citation en allemand erronée (dommage) : un génitif n'est pas marqué, et au lieu de 'Stummes Niederfallen fernen Sterne', il faut lire 'Stummes Niederfallen ferner Sterne' (chute silencieuse d'étoiles lointaines).

Anastasia Vinogradova (Université de Provence) traite de la traduction de poètes français tels que Baudelaire ou Mallarmé par des poètes symbolistes russes, une façon de traduire qui 'russifie' sensiblement les poèmes, et elle propose l'idée que ces 'infidélités' sont en fait systématiques et découlent d'une même logique correspondant à la vision du monde plus pessimiste et plus tragique d'une école de pensée qui s'oppose donc à son modèle en même temps qu'elle se réclame de son influence.

\section{Questions de linguistique :}

Gilles Bardy (Université de Provence) compare les onomatopées du français et du roumain, qu'il définit comme devant "produire un effet de sens à valeur évocatrice " (p. 257). Les énumérations sont plaisantes, et le lecteur ignorant du roumain sera intéressé d'apprendre qu'à notre 'atchoum' correspond 'hapciu', que le Roumain assoiffé fait gâl, gâl, gâl, et le corbeau câr, câr. Mais une particularité notable est que " la plupart des onomatopées roumaines peuvent remplir le rôle d'un verbe " (p. 259). La langue française est-elle pour autant dépourvue de moyens pour rendre cette particularité ? Le lecteur en jugera par lui-même. 
Kira Peshkova (Université de Provence) propose une modélisation de la terminologie des droits de l'homme en français et en russe, selon une méthode implémentable en informatique et en intelligence artificielle; dans cette démarche paradoxale (et pour laquelle la notion même de terme n'est pas définie), l'auteur semble estimer que l'on peut modéliser un domaine technique sans le maîtriser (les seules ressources juridiques de sa bibliographie sont des lexiques francorusses). Allant à l'encontre du postulat whorfien exprimé dans le 'Prélude', elle tire la possibilité de modéliser les structures conceptuelles du fait que " certains auteurs considèrent que les notions existent indépendamment de la langue dans laquelle elles sont réalisées, qu'elles décrivent de manière objective un domaine spécialisé et sont communes donc aux terminologies russe et française " (p. 268). Cette modélisation permettra alors de savoir de quoi on parle : " le modèle élaboré des Droits de l'Homme peut servir de base à l'étude approfondie des notions et de leur dénomination " (p. 275). On y part donc du principe qu'une étiquette identique dans les deux langues recouvre automatiquement un même concept, et parmi les notions traitées, il n'est pas fait de distinction entre celles qui renvoient à des institutions (Cour Européenne des Droits de l'Homme) et celles qui renvoient à des principes juridiques.

Malina Ivanova-Stankova (Académie des Sciences de Bulgarie), dans une comparaison entre le français et le bulgare, suppose peut-être un peu vite comme bien connu " qu'il existe un grand nombre de similitudes entre la sémantique et la fonction des temps passés en français et en bulgare " (p. 277). Parlant plus particulièrement de l'aoriste perfectif bulgare (temps 'passé fermé' qui peut souvent se rendre par un passé simple ou composé), elle montre par exemple qu'une succession de verbes ayant cette forme peut renvoyer à une suite d'événements dont l'antériorité au verbe introducteur nécessite le recours à un plus-que-parfait dans la traduction. Elle montre aussi que l'ordre des procès à cette forme ne reflète pas nécessairement la chronologie, ce qui semblerait la rapprocher davantage du prétérit anglais que du passé simple français (cf. l'exemple bien connu John fell. Jack pushed him).

Charles Zaremba (Université de Provence) : reprenant sa proposition du " Prélude " selon laquelle chaque langue se caractérise par une conceptualisation originale du monde (p. 285), l'auteur aborde deux différences sensibles entre le hongrois et le français : l'absence de genre en hongrois et son système verbal relativement pauvre comparé au français. II illustre ainsi de quelques exemples les acrobaties linguistiques auxquelles le traducteur vers le français doit se livrer dans un récit particulier où le sexe d'un personnage ne devrait pas être révélé trop tôt au lecteur. II en conclut que ces différences qui constituent l'obstacle principal à la traduction sont aussi l'occasion pour le traducteur d'être créatif.

En conclusion, c'est bien la variété qui caractérise cet ouvrage, et devant une telle abondance de propos, d'approches et de sujets différents, et nonobstant les réserves exprimées plus haut, il est plus que probable que tout lecteur, quelles que soient ses préoccupations, trouvera matière à nourrir sa réflexion. Ainsi par exemple, le linguiste contrastiviste trouvera sans doute 
autant d'intérêt aux particularités grammaticales de telle ou telle langue qu'aux nombreux rapprochements possibles entre systèmes, tandis que le spécialiste de la traduction pourra faire son profit de la profusion d'exemples et d'anecdotes, qui enrichissent la réflexion théorique.

\section{Yvon Keromnes, ATILF Université Paul Verlaine-Metz \& CNRS}

Traduire : un art de la contrainte

sous la direction de Charles Zaremba et Noël Dutrait,

Presses Universitaires de Provence, 2010.

ISBN 978-2-85399-750-8. 296 pages, prix : $26 €$. 